\title{
LOW - BETA SC QUARTER WAVE RESONATOR AND CRYOMODULE FOR SPIRAL 2
}

\author{
P.-E. Bernaudin, P. Bosland, S. Chel, G. Devanz, Ph. Hardy, F. Michel, A. Mosnier \\ CEA-Saclay, DSM/DAPNIA, 91191 - Gif Sur Yvette Cedex, FRANCE
}

\begin{abstract}
In the framework of the SPIRAL 2 project to be built in GANIL, we studied a complete cryomodule for the low beta (0.07) superconducting linac. The $88 \mathrm{MHz}$ quarter wave resonators were optimised for RF, mechanical and thermal considerations. A prototype resonator, which differs from the final resonator by the Helium tank and the removable end-plate geometry and material, has been built. We present the equipments developped for the preparation of this prototype as well as the results of the RF tests. We also set out in detail the mechanical calculation and measurements of the tuning system, and the main aspects of the cryomodule designed to save longitudinal space.
\end{abstract}

\section{INTRODUCTION}

In the framework of the SPIRAL 2 project [1], we designed the cryomodule of the low energy part of the superconducting linac. It is designed to house one $88 \mathrm{MHz} \beta=0.07$ quarter-wave resonator. Twelve of such a cryomodule should be inserted between the RFQ and the higher energy part of the superconducting linac.

The work is divided in three parts (resonator, prototype and test, complete cryomodule) and is summarised in the next sections, and in particular the mechanical calculations needed for the design of the whole cryomodule. In the meantime, a resonator prototype has been fabricated by ACCEL, and dedicated set-ups for preparation (chemical treatment, high pressure rinsing, and handling) of this resonator have been built. The results of the first measurements are presented.

\section{RESONATOR STUDY}

The main specifications of the resonator are:

- Frequency: $88 \mathrm{MHz}$ and $\beta$ optimal: 0.07

- Operating gradient $(\mathrm{Vacc} / \beta \lambda): 6.5 \mathrm{MV} / \mathrm{m}$

- Max losses at $6.5 \mathrm{MV} / \mathrm{m}: 7 \mathrm{~W}$

- $\mathrm{E}_{\text {peak }} / \mathrm{E}_{\text {acc }}<5$

- $\mathrm{B}_{\text {peak }} / \mathrm{E}_{\text {acc }}<10 \mathrm{mT} /(\mathrm{MV} / \mathrm{m})$

- Qext optimal (deuterons) $=6.710^{5}(\mathrm{bw}=130 \mathrm{~Hz})$

- Df/DPLHe $<13 \mathrm{~Hz} / \mathrm{mbar}$

- Frequency tuning range $: \pm 25 \mathrm{kHz}$

RF calculations were performed using Ansoft HFSS v8.5 and Vector Fields SOPRANO v8.7. Since the nominal accelerating field is high, the main purpose of the optimisation is to reduce the peak fields over accelerating field ratios. The cavity shape we selected (conical stem, rounded drift tubes, rounded top, removable bottom plate) seems the best compromise in consideration of the specifications and the ease of chemical treatment and rinsing operations. We chose to keep the beam axis position low enough to minimize the $\mathrm{B}_{\mathrm{x}}$ field component (in order to reduce the kick effect).

Table 1: Resonator parameters.

\begin{tabular}{|l|l|}
\hline Frequency & $88 \mathrm{MHz}$ \\
\hline Optimal $\beta$ & 0.07 \\
\hline $\mathrm{E}_{\text {peak }} / \mathrm{E}_{\text {acc }}$ & 5.00 \\
\hline $\mathrm{B}_{\text {peak }} / \mathrm{E}_{\text {acc }}$ & $8.75 \mathrm{mT} /(\mathrm{MV} / \mathrm{m})$ \\
\hline $\mathrm{R} / \mathrm{Q}$ & $632 \mathrm{Ohms}$ \\
\hline Quality factor $\mathrm{Q}_{0}$ & $2.210^{9}$ \\
\hline Cavity losses $\left.@ \mathrm{E}_{\mathrm{acc}}=6,5 \mathrm{MV} / \mathrm{m}\right)$ & $1.75 \mathrm{~W}$ \\
\hline Gasket losses $\left(@, \mathrm{E}_{\mathrm{acc}}=6,5 \mathrm{MV} / \mathrm{m}\right)$ & $50 \mathrm{~mW}$ \\
\hline Total height & $964.25 \mathrm{~mm}$ \\
\hline Diameter & $230 \mathrm{~mm}$ \\
\hline
\end{tabular}

The coaxial RF power coupler is connected to the bottom plate in vertical position (no additional port through Helium tank, low magnetic field area). With a 36 $\mathrm{mm}$ diameter $50 \Omega$ coupler, which is positioned $20 \mathrm{~mm}$ away from the cavity axis, and with an antenna penetration inside the cavity of $10 \mathrm{~mm}$, the wanted Qex for deuterons can be achieved. Multipactor computations using MUPAC [2], showed no multipacting barrier whatsoever in the 0 to $20 \mathrm{~kW}$ range in travelling wave mode.

Mechanical computations were performed using CEA CASTEM and EDS IDEAS codes. When both RF and mechanical computations were necessary (e.g. for helium pressure sensibility and tuning system calculations), deformed meshes have been generated using the mechanical codes and then transferred using the IDEAS "universal" format into the SOPRANO RF code. This method allows one to solve full $3 \mathrm{D}$ mechanics/RF coupled problems.

An alternative method using a reference RF computation as a basis for Slater perturbation method computations was applied in the CASTEM mechanical code on deformed meshes. For a cavity made out of bulk niobium, the optimisation of wall thickness with respect to helium pressure and tuning sensitivity results in a $4 \mathrm{~mm}$ niobium thickness. A strong requirement is the use of a stainless steel helium tank, which implies the use of a stainless steel flange for the removable bottom plate. Its shape is optimised to take into account several conflicting factors (qualification test at 2.6 bars, gasket in a low magnetic field area in order to reduce the RF losses, position of coupler to minimize its penetration in the cavity, mechanical behaviour due to thermal shocks during cool down and brazing operations). The best compromise between mechanics and RF aspects results in the ' $\mathrm{S}$ ' shape (Fig. 1). In the cryomodule, the bottom plate is not directly cooled by LHe. For this reason, it is made 
of copper with niobium coating to improve thermal conductivity while keeping losses at a low level.

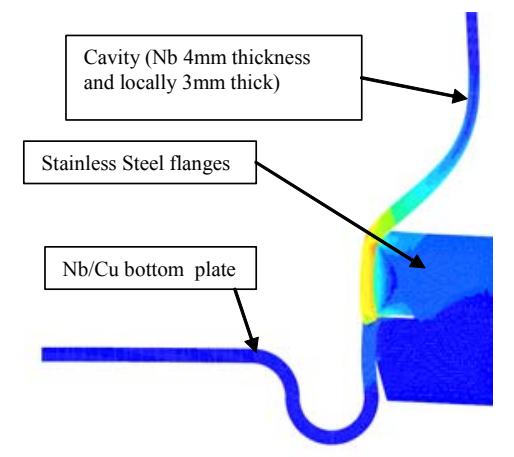

Figure 1: Von Mises stress and deformation in the bottom plate after a thermal shock at $70 \mathrm{~K}$.

The sensitivity of the cavity to helium pressure variations has been checked. Assuming a pressure stability of 1 mbar, the goal is to reach a sensitivity of $-13 \mathrm{~Hz} / \mathrm{mbar}$ in order to stay in a $10 \%$ bandwidth range. The pressure effects induce not only a vertical displacement and a deformation of the stem and toroidal top assembly into the cavity, but also a horizontal displacement and deformation of the half drift tubes into the cavity. If one considers a bare cavity, the first component is the main one (about 80\%). Therefore, several mechanical stiffeners have been studied and the most efficient one is able to decrease the pressure sensitivity as low as $-1.0 \mathrm{~Hz} / \mathrm{mbar}$ if necessary.

Considering the very small longitudinal space available, it has been decided not to tune the cavity using the beam tubes, but by deforming it in the direction perpendicular to the beam axis. An optimal height of the applicator has been found by numerical simulation. The shape of this applicator (Fig. 2) has also been optimised in order to decrease stress while keeping the tuning sensitivity as high as possible.
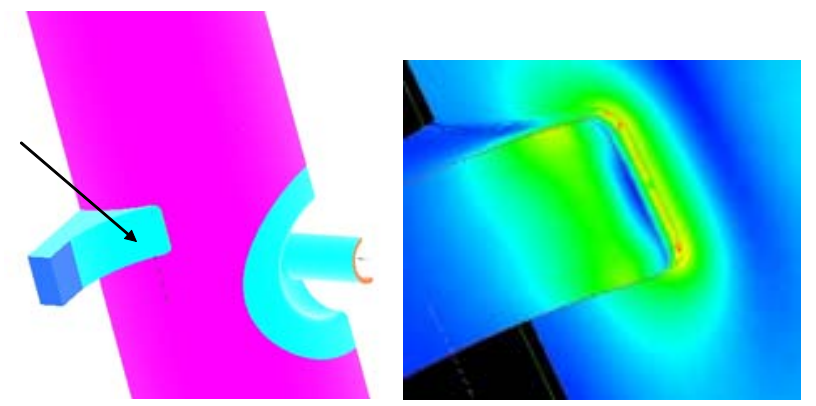

Figure 2: Tuner applicator (arrow) welded on the cavity outer cylinder (left) and stress distribution (right).

The tuning range is limited by mechanical stress (400 $\mathrm{MPa}$ at $4 \mathrm{~K}$ ). It is optimized up to $\pm 24.2 \mathrm{kHz}$ by changing the cavity walls thickness from $3 \mathrm{~mm}$ to $4 \mathrm{~mm}$.

Modal computations showed that microphonics are a potential cause of trouble. The lower mechanical resonance frequency is around $60 \mathrm{~Hz}$ and this frequency cannot be raised significantly whichever stiffener is used.
If it appears necessary, a mechanical damper shall be installed inside the stem [3].

\section{PROTOTYPE AND TEST RESULTS}

The main objective of the test in vertical cryostat is to verify that the specified accelerating gradient and cavity losses are achievable. The design of this prototype is slightly different from the resonator presented in previous section since the Helium tank and the tuning system applicators are not included, and the bottom plate and flanges are made of NbTi alloy. This prototype (Fig. 3) has been built by ACCEL, and after delivery at Saclay, the measured frequency at room temperature and atmospheric pressure is $87.62 \mathrm{MHz}$.
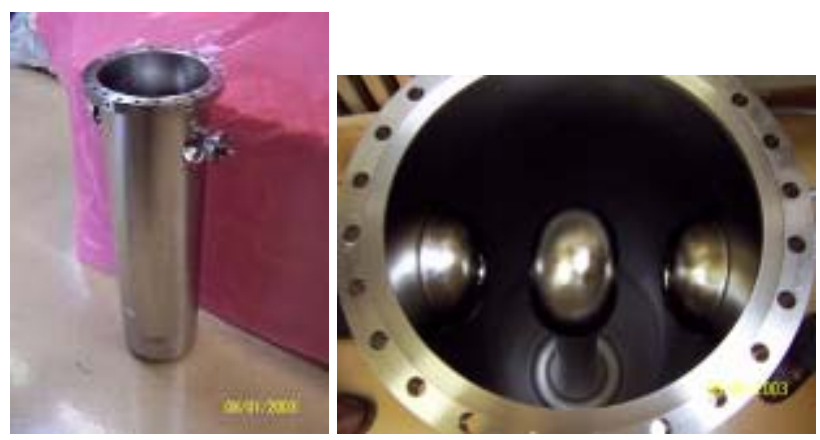

Figure 3: Cavity prototype (outer and internal views).

Before RF tests, cavity was rinsed in ultrasonic bath, then the inner surface of the cavity has been chemically treated with the FNP 1-1-2 acid mixture circulating inside the cavity. The injection of acid in the cavity is performed at two different heights in order to prevent the stagnation of acid and bubbles. With the pumps at a flow rate of 20 liters/minute, which determines the filling-up time of the cavity volume of $1 \mathrm{~min} 50 \mathrm{~s}$. About one hour was needed for a complete cycle of 185 microns.

Moreover, a set-up has been developed in order to perform a high pressure rinsing (HPR) of the cavity surface with ultra-pure water $(>18 \mathrm{MOhms})$ in clean room, a necessary treatment if one wants to obtain the specified performances. Due to the complex shape of such a cavity, the possibility of rinsing led us to consider a removable bottom plate in order to get a better access to the cavity volume. The HPR set-up is nearly horizontal. The nozzle is kept still while the cavity rotates and translates. The geometry of the nozzle is a hemispherical piece drilled with nine $0.6 \mathrm{~mm}$ channels spaced by $30^{\circ}$. In order to rinse the whole cavity surface, 1800 rotations are needed (total duration of 3 hours).

After cavity preparation, measurements were performed at $4.21 \mathrm{~K}$. The measured frequency of $87.788 \mathrm{MHz}$ is in very good agreement with frequency shift calculations. Qo above $10^{9}$ was reached at the nominal Eacc of 6.5 MV/m (equivalent to $3.5 \mathrm{~W}$ of RF losses). Most of these losses as well as the slope of the curve and the maximum Eacc value are due to a special Indium + Copper gasket of the bottom flange. We prepare a new test with a superconducting gasket to discriminate 
between cavity and gasket losses, and we have already designed a flange arrangement with cooling channels for the final resonators.

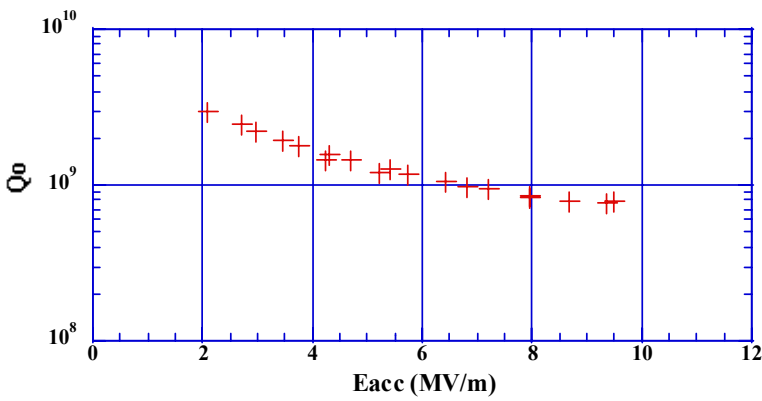

Figure 4: Q vs Eacc curve of the QWR prototype.

\section{COMPLETE CRYOMODULE}

Each cryomodule contains a single cavity (Fig. 5). This choice eases conception, maintenance and alignment tasks. The cavity vacuum and the isolation vacuum are separated. The cryomodule is box-shaped, with one door on each side for easy access. This helps the clean room assembly, the assembly of the very short beam tubes extremities, of the tuning system, etc. The $70 \mathrm{~K}$ thermal screen has the same box shape, and is made of three separated, bolted parts. Clean room assembly includes the cavity, power coupler, beam tube extremities and bellows, end valves and positioning of all these elements inside the opened cryomodule.

Special care has been taken to keep the distance between cavities as low as possible, as required by beam dynamics studies. To do so, the bellows and beam tube between $4 \mathrm{~K}$ and $300 \mathrm{~K}$ have been kept as short as afforded by the cryogenic system, the number of welding has been reduced, and flanges geometry adapted to gain space.

The thermal load on the $70 \mathrm{~K}$ copper shield is around $25 \mathrm{~W}$ ( $1 \mathrm{~W}$ through the beam tube), and the load on the $4 \mathrm{~K}$ components is around $15 \mathrm{~W}$ (assuming $1.3 \mathrm{~W}$ from the power coupler). Elements not in contact with liquid helium, like the bottom plate and the coupler port, are connected to a small liquid helium reservoir located beneath the cavity through copper breads. The tuning system is based on the SOLEIL \& Super-3HC design [4]. The design allows one to place the stepping motor either inside the cryomodule or outside. The gear box will be kept inside the cryomodule in both cases.

The cavity will be supported and fixed inside the cryomodule by ten Ta6V rods. Two vertical rods support the cavity, and eight rods placed in X pattern (four at the top and four at the bottom) suppress any horizontal movements of the cavity during cool down. Due to the thermal shrinking of the vertical $\mathrm{Ta} 6 \mathrm{~V}$ rods combined with the shrinking of the stainless steel helium tank and that of the cavity niobium, the cavity beam tubes will move up by about $1.1 \mathrm{~mm}$ during cool down.
During the cryomodule is assembled, alignment of the cavity inside the cryostat is performed by using the Ta6V rods. As the cavity is equipped with external references of his axis, it will be possible to perform the alignment of this cavity inside the linac tunel at room temperature by moving its whole cryomodule with respect to the other ones. Measurements of the cavity beam axis displacement during cool down is foreseen, mainly to check the validity of calculations and to improve, if necessary, the alignment procedures.

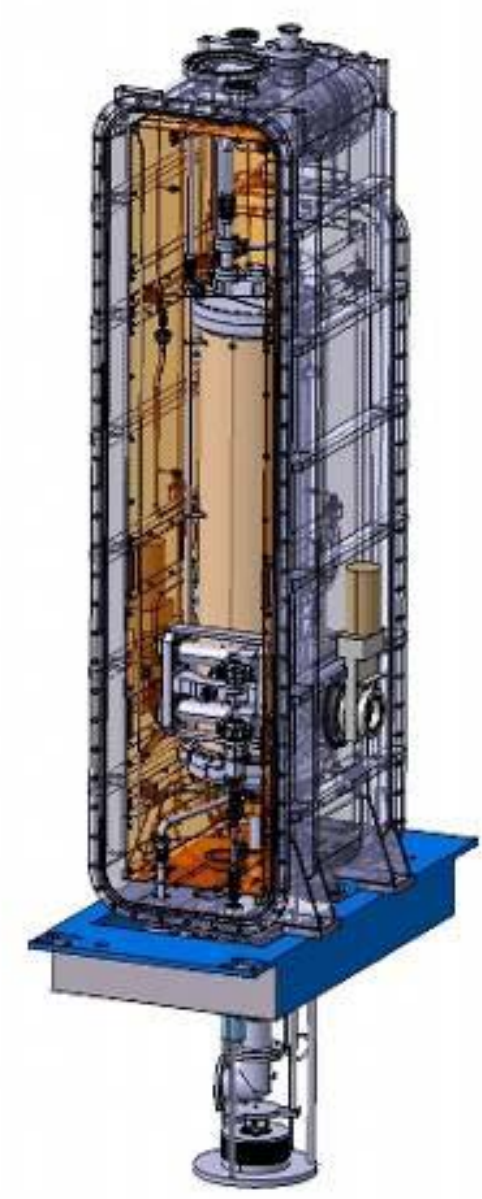

Figure 5: QWR cavity housed in his cryomodule.

\section{REFERENCES}

[1] A. Mosnier, "SPIRAL2 : A High Intensity Deuteron and Ion Linear Accelerator for Exotic Beam Production", PAC 2003, Portland

[2] G. Devanz, "Multipactor simulations in sc cavities and power couplers", Phys. Rev. ST Accel. Beams 4, 012001 (2001)

[3] A. Facco \& V. Zviagintsev, "Mechanical stabilisation of superconducting quarter wave resonators", Proceedings of the PAC-97, Vancouver, vol. 3

[4] S. Chel et al., "SUPER 3HC cryomodule: layout and first tests", Proceedings of the EPAC-0, Paris, France 